\title{
Corrections to Compact Nonlinear Model of an Implantable Electrode Array for Spinal Cord Stimulation
}

\author{
Sinduja Seshadri and Jonathan Scott, Senior Member, IEEE
}

Reference [1] describes the construction of a model of the electrode-electrolyte interface and the surrounding electrolyte for a platinum-electrode array used in Spinal Cord Stimulation (SCS) applications. In that paper, a key advancement was a compact equivalent-circuit model of a constant phase element (CPE) that can be entered into the SPICE simulator. The authors of [1] relied heavily on mathematics taken from [2] by Morrison. Fig 11 reproduced from Fig.4 in [1] depicts the potentially infinite equivalent circuit used to represent the lumped approximation of a CPE. This circuit represents an impedance that is an approximation to the impedance that would be presented by a CPE. The authors of [1] are faced with finding $C, R, m$ and $k$ to approximate a CPE whose magnitude is $\left|Z_{C P E}\right|$ at some frequency $\omega$ and whose phase is $\theta_{C P E}$.

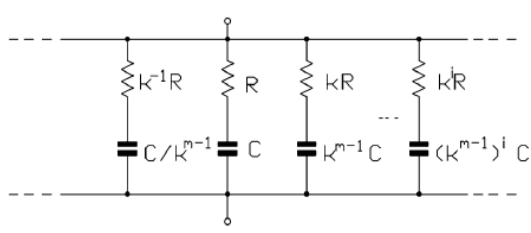

Fig. 1. The theoretically infinite lumped-RC approximation of a CPE reproduced from [1].

The value of $m$ is obtained from (8) in [1] which is derived from (50) in [2]:

$$
m=\frac{\pi}{2 \theta_{C P E}}
$$

Morrison's accuracy parameter, $k$, is chosen arbitrarily while values of $R$ and $C$ are obtained using:

$$
\left|Y_{\theta}^{\prime}\right| \approx \frac{1}{\left|Z_{C P E}\right|} \approx \frac{(\omega R C)^{\frac{1}{m}}}{R} y_{\theta}
$$

where $\left|Y_{\theta}^{\prime}\right|$ is the magnitude of the approximation and

$$
y_{\theta}=\frac{\pi}{m \ln k} \sec \frac{1}{2} \pi\left(1-\frac{2}{m}\right),
$$

where $\omega$ is the radian angular frequency.

The exact value of $\mathrm{Y}_{\theta}$ is expressed as an infinite series, with $R=C=1$. After a "tedious amount of algebra", Morrison arrived at (4) reproduced from (39) in [2]:

$$
Y_{\theta}=\frac{(j \omega)^{1 / m} \pi}{\alpha}\left\{\sum_{r=1}^{\infty} \frac{1}{c}(a-b j)\right\}+y_{\theta}(j \omega)^{1 / m},
$$

where $j$ is the imaginary number,

$$
\alpha=\frac{1}{2} m \ln k
$$

$a, b$ and $c$ contain several nested trigonometric and hyperbolic functions.

Morrison considered the analytical solution of $Y_{\theta}$ intractable. Being interested in the phase of the CPE network rather than its magnitude, he proceeded to make some assumptions. The assumption is (40) from [2]: if

$$
1 \ll \cosh \left(\frac{1}{2} \pi^{2} \alpha^{-1}\right) \approx \sinh \left(\frac{1}{2} \pi^{2} \alpha^{-1}\right),
$$

then, the sine and cosine terms in $a$ and $b$ become very small and ignoring the higher harmonics, (4) can be written as (41) from [2]:

$$
\begin{array}{r}
Y_{\theta} \approx \frac{(j \omega)^{1 / m} \pi}{\alpha} \frac{\cosh \left(\frac{1}{2} \pi^{2} \alpha^{-1}\right)}{\cosh \left(\pi^{2} \alpha^{-1}\right)} \\
\cdot\left[\cos \left(2 \pi x-\frac{1}{2} \pi p\right)-j \sin \left(2 \pi x-\frac{1}{2} \pi p\right)\right] \\
+\frac{\pi}{2 \alpha}(j \omega)^{1 / m} \sec \left(\frac{1}{2} p \pi\right),
\end{array}
$$

where

$$
x=\frac{\ln \omega}{m \ln k}, p=1-\frac{2}{m} .
$$

Morrison concluded that the magnitude of $Y_{\theta}$ is not affected by the cosine hyperbolic function in $(7)$, so $\left|Y_{\theta}\right| \approx y_{\theta}(\omega)^{1 / m}$ where $y_{\theta}$ is given in (3). This allowed him to sidestep the infinite summation in (4) and formulate the admittance given in its most general form in (2). Then, he developed the equations to produce the phase of a CPE network which are (6) and (7) in [1] reproduced from (50) and (51) in [2]:

$$
\angle Y_{\theta}^{\prime}=\frac{\pi}{2 m}-\theta_{p} \sin \left[\frac{2 \pi \ln \omega R C}{m \ln k}-\frac{1}{2} \pi\left(1-\frac{2}{m}\right)\right],
$$

where $\angle Y_{\theta}^{\prime}$ is the argument of the approximation $Y_{\theta}^{\prime}$, and

$$
\theta_{p} \approx \frac{m \ln k}{\pi} \frac{\cosh \frac{\pi^{2}}{m \ln k}}{\cosh \frac{2 \pi^{2}}{m \ln k}} \cos \frac{1}{2} \pi\left(1-\frac{2}{m}\right) .
$$

The error in phase computed from 9 and $(10)$ can be made sufficiently small by choosing a value for $k$ sufficiently close to 1. While the mathematics in [2] produces a zero error in phase, it results in a finite error in magnitude. Morrison makes no claim about this error in the magnitude approximation, which escaped the authors of $[1]$.

We performed a literature review using the papers that cited [2] and [1] as the basis of our search. Lerner mentions 
Morrison's magnitude of admittance error but does not provide any further details [3]. Several papers used the mathematics of [2] for determining phase and others cited [1] and [2] for the CPE model.

This manuscript proposes a correction to solve the problem of the magnitude error and other errors in [2]. The algorithm from [1] can be executed in its current form with an addition. The error between the desired CPE magnitude $\left|Z_{C P E}\right|_{\omega}$, and the magnitude returned by the algorithm $|Z|_{\omega}$, does not change with frequency. We introduce a constant correction factor $\beta$,

$$
\beta=\frac{\left|Z_{C P E}\right|_{\omega}}{\left|Z_{\omega}\right|}
$$

and modify $Z_{\omega}$ derived from Fig 1

$$
Z_{\omega: f \rightarrow 0}=\left(\frac{1}{Z_{R C}}+\frac{1}{Z_{\left(R k^{i}\right)\left(C\left(k^{(m-1)}\right)^{i}\right)}}\right)^{-1}
$$

and

$$
Z_{\omega: f \rightarrow \infty}=\left(\frac{1}{Z_{R C}}+\frac{1}{Z_{\left(R k^{-i}\right)\left(C\left(k^{(m-1)}\right)^{-i}\right)}}\right)^{-1} .
$$

The counter $i$ ranges such that the time constant of the first and last branch span the required bandwidth, $f$.

The modified algorithm was tested on Morrison's $45^{\circ}$ network example in [2]. The parameters are:

$$
k=6.25, m=2,\left|Z_{C P E}\right|_{\omega_{0}}=10.5 \mathrm{k} \Omega,
$$

where

$$
f=1.59 \mathrm{kHz}: \omega_{0}=10,000 \mathrm{rad} / \mathrm{sec} .
$$

The magnitude of output impedance computed from (12) and (13) is

$$
|Z|_{\omega_{0}}=9.4 \mathrm{k} \Omega
$$

and using (11) gives

$$
\beta=1.12 \text {. }
$$

Morrison's original result is shown in Fig 2 and the scaled result is shown in Fig 3 ,

In Morrison's numerical example, he replaces the last $\mathrm{RC}$ branch at either end with a capacitor and a resistor as in Fig 2 . He states that the effect of adding more elements to the right and left of the branches is equivalent to adding one lumped resistor or capacitor to the network [2].

Morrison calculates the values of this resistor and capacitor by considering the proper geometric series of these elements [2]. He used (52) and (54) in [2] to calculate the value for the terminating resistor and capacitor respectively, reproduced here as (18) and (19),

$$
\begin{aligned}
& Y_{T}=\frac{10^{-3}}{390}\left[1+\frac{1}{k}+\frac{1}{k^{2}}+\ldots\right], \\
& C_{T}=260\left[\frac{k}{k-1}\right]=310 \mu \mu \mathrm{F} .
\end{aligned}
$$

The values $390 \mathrm{k} \Omega$ and $260 \mathrm{pF}$ are the resistor or capacitor values of the last branches in the network. According to Fig, 2 , the terminating resistor and capacitor values are $327 \mathrm{k} \Omega$ and $310 \mathrm{pF}$. Morrison does not explain how he arrived at (18), and (19) proves to be incorrect.
The terminating resistor and capacitor values are a product of infinite sums and are given by,

$$
Y_{T}=\frac{1}{R_{F}} \sum_{i=0}^{\infty}\left(\frac{1}{k}\right)^{i}
$$

and

$$
C_{T}=C_{F} \sum_{i=1}^{\infty}\left(\left(k^{-(m-1)}\right)^{i}\right),
$$

where $R_{F}$ and $C_{F}$ are the resistor and capacitor values in the last RC branch. Looking at 21, it is obvious that (19), Morrison's (54), is wrong because $m$ does not appear.

The geometric series given in (20) and (21) are convergent. (20) can be written as

$$
Y_{T}=\frac{1}{Z_{R_{F} C_{F}}}\left[\frac{1}{k-1}\right] ; Z_{T}=\frac{1}{Y_{T}},
$$

where $Z_{R_{F} C_{F}}$ is the impedance of the last branch. The correct converged formula for (21) is

$$
C_{T}=C_{F}\left[\frac{k}{k^{m}-k}\right] \text {. }
$$

In Morrison's example, the original branch resistor and capacitor values are $125 \mathrm{k} \Omega$ and $253 \mathrm{pF}$. The terminating resistor and capacitor values are thus $656 \mathrm{k} \Omega$ and $48 \mathrm{pF}$ as shown in Fig 3

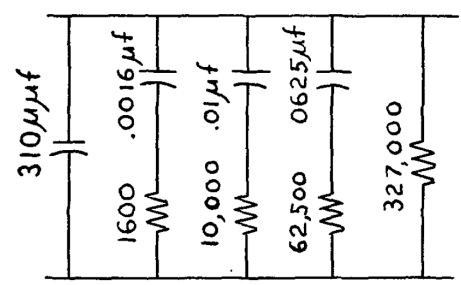

Fig. 2. Morrison's finite network of the numerical example in [2].

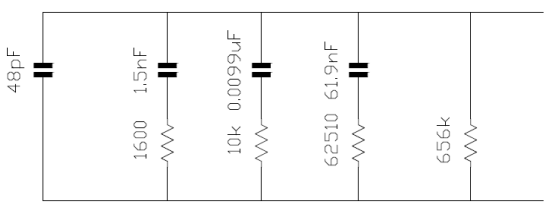

Fig. 3. Revised network for Morrison's numerical example.

The values used in Morrison's original example were very approximate and yielded incorrect results except for $\angle Y_{\theta}$. We refer interested readers to the website: https://goo.gl/VBsKc9, for the Python code that generates the CPE network and a SPICE subcircuit.

\section{REFERENCES}

[1] J.Scott and P.Single, "Compact nonlinear model of an implantable electrode array for spinal cord stimulation (SCS)," IEEE Transactions on Biomedical Circuits and Systems, vol. 8, pp. 382-290, Jun. 2014.

[2] R.Morrison, "RC constant-argument driving-point admittances," IRE Transactions on Circuit Theory, vol. 6, pp. 310-317, Sep. 1959.

[3] R.Lerner, "The design of a constant-angle or power-law magnitude impedance," IEEE Transactions on Circuit Theory, vol. 10, pp. 98-107, Mar. 1963. 


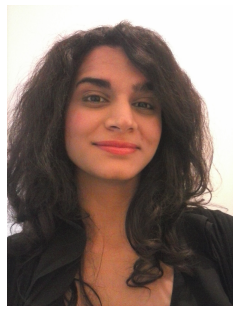

Sinduja Seshadri received the BSc. Physics degree from University of Waikato, Hamilton, New Zealand in 2017 , where she is currently pursuing a researchbased Master's in Electronic Engineering.

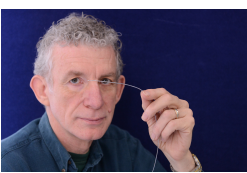

Jonathan Scott (M'80-SM'99) holds five degrees and is the Foundation Professor in Electronic Engineering at the University of Waikato, Hamilton, New Zealand.

From 1998 to 2006, he was with Hewlett-Packard, now Agilent Technologies, Microwave Technology Center, Santa Rosa, CA, USA, where he was responsible for advanced measurement systems. In 1997 and 1998, he was Chief Engineer at RF Technology in Sydney. He was with the Department of Electrical Engineering, University of Sydney, prior to 1997. He has authored over 100 refereed publications, several book chapters and a textbook, and he holds a dozen patents, several covering active products. His educational interests include threshold concepts and their application, particularly across engineering disciplines. 